Historic, Archive Document

Do not assume content reflects current scientific knowledge, policies, or practices. 



\section{GARDEN SEEDS}

At these low prices we prepay postage on all Garden Seeds in quantities listed.

\section{BEANS}

Black Wax Pencil Pod

Postpaid

Pkt. Per lb.

Golden Wax

$$
\$ .10 \$ .40
$$

.10

Curry's Rust Proof

.10

.40

Bountiful

.10

Davis White Wax

.10

Burpee's Stringless Green Pod

Giant Stringless Green Pod

Wardwell's Kidney Wax

Tenn. Green Pod

Early Six Weeks

Red Valentine

Kentucky Wonder

Kentucky Wonder Wax

Cutshort

Lazy Wife

White Creasback

White Dutch Caseknife

Burpee's Bush Lima

Henderson's Bush Lima

Large White Lima

White Navy

.10

.10

.10

.10

.10

.10

.10

.10

.10

.10

.10

.10

.10

.10

.10

.10

\section{BEETS}

Extra Early Egyptian $\quad \$$ Pkt.

Crosby Egyptian _._._._. $\quad .10$

Detroit Dark Red …- $\quad .05$

Early Blood Turnip _-_._. $\quad .05$

Eclipse

.05

Long Dark Blood

.05

$\begin{array}{rr}\text { oz. } & \text { 1/4 lb. } \\ \$ 10 & \$ .35 \\ .15 & .35 \\ .10 & .35 \\ .10 & .35 \\ .10 & .35 \\ .10 & .35\end{array}$

$1 \mathrm{~b}$.

$\$ .90$

.85

.90

.85

.85

.80

\section{STOCK AND SUGAR BEETS}

Mammoth Long Red Mangel $\$$ oz. $\$^{1 / 4} \mathbf{l b}$.

Golden Tankard Mangel _-..-. $\quad .10$

White Klein Wanzleben Sugar _._. $\quad .10$

Giant Half Sugar

.10

\section{SWISS CHARD}

Giant Lucullus

$\$ .05 \quad \$ .10 \quad \$ .35 \quad \$ 1.00$

\section{CABBAGE}

All Head Early

Pkt.

Early Jersey Wakefield --- .10

oz.

Copenhagen Market

.10

$\$ .25$

$\$ 1 / 4 \mathrm{lb}$.

.25

.30

.75

.85

Early Dwarf Flat Dutch _. $\quad .10$

.25

Premium Late Flat Dutch_. $\quad .10$

.25

Large Late Drumhead

.10

.25

All Season

.25

Danish Ball Head

.10

.30

.25

.40

.75

.75

.75

.75

1.00

.70

Mam. Red Rock

.10

.10

.90

$1 \mathrm{~b}$.

$\$ 2.25$

2.00

2.75

2.25

2.25

2.25

2.25

3.00

\section{CARROTS}

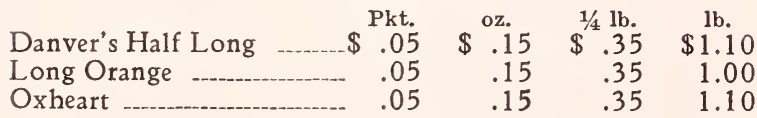

White Plume

CELERY

Golden Self Blanching

$\$ .10$

.10

$\$ .25$

$1 / 4 \mathrm{lb}$.

$\$ .85$

1.25

COLLARDS

True Georgia $\$ .05$

$\$ .15$

$\$ .30$

\section{SWEET CORN}

Golden Bantam $\$ .10$

Early Minnesota .10

Stowell's Evergreen .10

Country Gentleman

.10

Early Adams

.10

Howling Mob

.10

lb.

$10 \mathrm{lbs}$.

$\$ .30$

$\$ 2.00$

.30

.30

2.00

2.00

.30

2.00

1.65

.35

2.50

\section{CUCUMBERS}

Early Fortune $\$$ Pkt.

Davis Perfect

$\$ .20$

Early Cluster

.05

.15

Everbearing

.05

.15

Japanese Climbing

.05

.15

Chicago Pickling

.10

Long Green

.05

West India Gherkin

.15

.15

.10

.25

$\$ 1 / 4.16$.

.50

.40

.50

.50

.50

.50

.50

EGG PLANT

New York Improved Purple

Pkt.

Black Beauty

$\$ .10$

$1 / 2$ oz.

$\$ .25$

.25

$1 \mathrm{~b}$.

$\$ 1.50$

1.50

1.00

1.25

1.50

1.25

1.50

1.75

$\$ \begin{aligned} & \text { oz. } \\ & .45 \\ & .45\end{aligned}$

ENDIVE

Green Curled

$\$ .05$

White Curled

$\begin{array}{rr}\text { ENDIVE } & \\ & \text { Pkt. } \\ & .05 \\ -05\end{array}$

$\$$ oz.

.15

$1 / 4 \mathrm{lb}$.

$\$ .40$

.40

KALE

Dwarf Curled Scotch

$\$$ Pkt.

Tall Curled Scotch

KOHL RABI

Early White Vienna

Early Purple Vienna

$$
\text { -- }
$$$$
.05
$$

$\$ \stackrel{\text { oz. }}{.15}$

.15

$\$ \begin{aligned} & 1 / 4 \mathrm{lb} \\ & \$ .50 \\ & 50\end{aligned}$

.50

\section{LETTUCE}

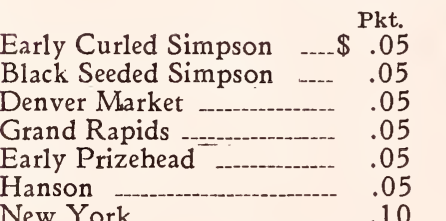

Pkt. $\$ .05$

oz.

$1 / 4 \mathrm{lb}$.

.15

.50

.50

\section{MUSKMELON}

Acme or Baltimore

Pkt.

Extra Early Hackensack - - . .05

Eden Gem _._______ .05

Edward's Perfecto

.10

Hale's Best

.10

Pollock's 10-25

.10

Rocky Ford

.05

Burrel's Gem

.10

$\$$ oz.

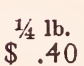

lb.

Paul Rose

.15

.15

.15

.15

.15

.30

.50

.50

.40

.40

.40

.65

1.25

1.25

1.50

1.25

1.25

1.25 


\section{Flower Seeds}

Alyssum, Little Gem

Asters, Queen of the Market, mixed 10

Asters, Giant, mixed

Bachelor's Button, mixed

Marigold, African, double mixed

Nasturtiums, Dwarf, mixed, 2 oz., $25 c$

Nasturtiums, Tall, mixed; $2 \mathrm{oz}$. $25 c$

Petunia, Single, mixed

Phlox, Drummondii, mixed

Sweet Peas, Spencer, mixed; 2 oz., $45 c$

Sweet Peas, Grandiflora, mixed 2 oz., $25 c$

Sweet William, double mixed

Zinnia, Double Giant, mixed
Pkt.

$\$ .10$

.10

.10

.10

.10

.10

.10

.10

.10

$1 / 4 \mathrm{oz}$.

$\$ .25$

.40

.40

.15

.20

.20

.75

$.40 \quad .25$

.25

.10

$\$ .10$

.10

$\$ .40$

.40

\section{Flower Plants}

Zinnia, Petunia, Phlox, Verbena

Salvia, and others

\section{CANNA BULBS}

Red Flowering, Tall Bronze Foliage Doz. Per 100

Mixed Colors, Tall

Fancy Named Varieties; dozen, $\$ 1.25$ to $\$ 2.50 ; 100$, $\$ 9.00$ to $\$ 18.00$.

\section{DAHLIA BULBS}

$$
\text { Each }
$$

Named Varieties

\section{GLADIOLI BULBS}

Finest Mixture $\$ .40 \$ 3.00$ BAMBOO Bulbs, each (postpaid) - .40

TUBEROSE BULBS

Mexican Everblooming

Doz. Per 100 $\$ .75 \$ \$ 4.50$

\section{Vegetable Plants}

All our plants are hardy, thrifty, trans-planted plants, and are well packed for shipping, thus insuring arrival in good condition. The prices quoted are postpaid.

Tomato, all varieties

Cabbage, all varieties

Āsk

Peppers, hot and sweet

Cauliflower for

Egg Plant

Sweet Potato

Prices

Bermuda Onion Plants

\section{SPRAYERS}

No. 3 Tin Intermittent G-V, pint

No. 4 Brass Intermittent $G-V$, pint

No. 5 Tin Intermittent $G-V$, quart

No. 6 Brass Intermittent G-V, quart

Hudson Misty, quart

Perfection Comp. Air, No. 110 Galv.

Perfection Comp. Air, No. 111 Brass

Hudson Jr. Comp. Air, No. 140

Hudson No. 4A Brass Pump

Hudson Dust Sprayer, No. 604

A Standard Bucket Pump Sprayer

Nozzles, Hose, Spray Poles

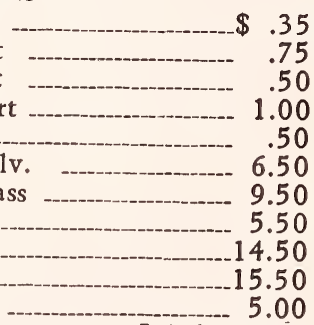

Ask for Prices 